\title{
GMR
}

\section{shRNA inhibits the expression of chicken telomerase reverse transcriptase in MDCC- MSB1 cells}

\author{
N. Jiang, X.B. Zheng, Z.Y. Zhao, Z.G. Qin and T.J. Liu \\ China-Japan Union Hospital, Jilin University, Changchun, Jilin, China \\ Corresponding author: T.J. Liu \\ E-mail: tianjiliu2015@sina.com \\ Genet. Mol. Res. 15 (1): gmr.15016979 \\ Received September 12, 2015 \\ Accepted November 24, 2015 \\ Published January 29, 2016 \\ DOI http://dx.doi.org/10.4238/gmr.15016979
}

\begin{abstract}
Here, we investigated the effects of blocking chicken telomerase reverse transcriptase (chTERT) in MDCC-MSB1 cells, using small-hairpin RNAs (shRNAs) to interfere with gene expression. shRNAs specific to chTERT mRNA were designed, cloned into DNA plasmid vectors, and transfected into MDCC-MSB1 cells. The transfected chTERT RNAs were expressed by the RNA polymerase machinery of the MDCC-MSB1 cells. mRNA expression in transfected MDCC-MSB1 cells was detected using real-time PCR. After transfection, telomerase activity was monitored via a modified telomeric repeat amplification protocol assay, and cell cycle analysis was performed using a flow cytometer. At $72 \mathrm{~h}$ after transfection, chTERT expression was considerably reduced in cells transfected with shRNA; the highest inhibition rate was $89 \%$. Compared with the control group, telomerase activity was significantly reduced and the cells failed to progress to $S$ phase. shRNA effectively reduced telomerase activity and prohibited the transition of MDCC-MSB1 cells from G2/M to $S$ phase.
\end{abstract}

Key words: Chicken telomerase reverse transcriptase; Real-time PCR; Small-hairpin RNA 


\section{INTRODUCTION}

High telomerase activity is observed in most tumors and immortalized cells, suggesting that telomerase activation plays an important role in tumorigenesis (Venkatesan and Price, 1998). Telomerase reverse transcriptase (TERT) is a critical factor in telomerase activation and represents a promising molecular target for tumor therapy (Delany and Daniels, 2004). Therefore, inhibition of tumor cell proliferation by the suppression of TERT mRNA expression is currently a hot topic in cancer research (Delecluse and Hammerschmidt, 1993; Swanberg et al., 2004).

Marek's disease (MD) is characterized by a malignant T-lymphoid tumor and is of particular interest to the poultry industry. MD in chickens can be controlled by vaccines based on non-pathogenic strains of the turkey herpes virus. The agent causing MD is an extremely contagious oncogenic herpes virus, the first one identified experimentally; however, its spread can be prevented by vaccination. The MD virus is now regarded as an ideal model for studying tumor initiation and progression (Woźniakowski and Niczyporuk, 2015).

RNA interference (RNAi) is an evolutionarily conserved mechanism that has been exploited experimentally to investigate the effects of silencing specific genes (Olejniczak et al., 2010). This method has great potential for use in targeted cancer therapy. Elucidation of the mechanism of RNAi led to the development of several novel RNAi-based therapeutic approaches that are currently in the early phases of clinical trials (Pecot et al., 2011). In RNAi, mRNA transcribed from the genome forms a duplex with an inserted double-stranded RNA (dsRNA) and is then degraded, diminishing or blocking expression of the transcribed gene (Peng et al., 2015). RNAi technology has considerable potential for application in many areas of biomedical research from exploring gene function to cancer treatments.

In the present study, small-hairpin RNAs (shRNAs) were designed to specifically target chicken TERT (chTERT) mRNAs. These shRNAs were transfected into MDCC-MSB1 cells with the aim of investigating expression of chTERT, telomerase activity, and cell cycles in cells undergoing oncogenic changes.

\section{MATERIAL AND METHODS}

\section{Cells and plasmids}

MDCC-MSB1 cells were purchased from the Institute of Veterinary Drug Control, China. The plasmid pSilencer 1.0-U6 was already available in this laboratory.

\section{Construction of pSi-U6 plasmid}

BLASTn was used to analyze the chTERT sequence, and shRNA sequences were designed based on the obtained information; the shRNA sequences were named sh-1, sh-2, and sh-3 (Table 1). Sense and anti-sense oligodeoxyribonucleotide sequences separated by TTCAAGAGA were synthesized to form a hairpin structure. An RNA polymerase terminal sequence TTTTTT was added to the 3 '-end of each anti-sense strand, and then adaptor sequences containing Apal (TaKaRa, Japan) and EcoRI (TaKaRa) restriction sites were added. The control sequences were the reverse sequences of sh-1 with no homogeneity to target genes. pSilencer 1.0-U6 was digested with Apal and $E c o R I$ and purified using agarose gel electrophoresis. An agarose gel extraction kit (Roche, 
Switzerland) was used to retrieve the long DNA fragments, which were stored at $-20^{\circ} \mathrm{C}$.

Synthesized DNA was diluted to $1.0 \mathrm{mg} / \mu \mathrm{L}$. A reaction mixture was prepared containing $2 \mu \mathrm{L}$ complementary and coding strand solutions in $46 \mu \mathrm{L}$ annealing buffer solution (100 mM potassium acetate, $30 \mathrm{mM}$ HEPES, $2 \mathrm{mM}$ magnesium acetate, $\mathrm{pH}$ 7.4). The mixture was incubated at $90^{\circ} \mathrm{C}$ for 3 min and then at $37^{\circ} \mathrm{C}$ for $1 \mathrm{~h}$ to anneal the complementary and coding strands. These dsDNAfragments were then ligated with vector pSilencer 1.0-U6 and transformed into Escherichia coli; a sample of the plasmid mixture was digested with HindIII (TaKaRa) and Xhol (TaKaRa) for confirmation. In order to detect the accuracy of the ligated fragments, positive plasmids were sequenced, and sequences named pSi-U6-Ctrl, pSi-U6-sh-1, pSi-U6-sh-2, and pSi-U6-sh-3 were obtained.

Table 1. shRNA sequences.
\begin{tabular}{l|l}
\hline shRNA name & Sequence \\
\hline sh-1(U) & GGTCTGGTTATCAGCCTTCTTCAAGAGAGAAGGCTGATAACCAGACCTTTTTT \\
\hline sh-1(C) & AATTAAAAAAGGTCTGGTTATCAGCCTTCTCTCTTGAAGAAGGCTGATAACCAGACCGGCC \\
\hline sh-2(U) & GCCATAACAAATGCCGGTTCTTCAAGAGAGAACCGGCATTTGTTATGGCTTTTTT \\
\hline sh-2(C) & AATTAAAAAAGCCATAACAAATGCCGGTTCTCTCTTGAAGAACCGGCATTTGTTATGGCGGCC \\
\hline sh-3(U) & GATCCCAAGGGATACTATGTTCAAGAGACATAGTATCCCTTGGGATCTTTTTT \\
\hline sh-3(C) & AATTAAAAAAGATCCCAAGGGATACTATGTCTCTTGAACATAGTATCCCTTGGGATCGGCC \\
\hline shCtrl(U) & CTTCCGACTATTGGTCTGGTTCAAGAGACCAGACCAATAGTCGGAAGTTTTTT \\
\hline shCtrl(C) & AATTAAAAAACTTCCGACTATTGGTCTGGTCTCTTGAACCAGACCAATAGTCGGAAGGGCC \\
\hline $\mathrm{U}=$ non-Coding strand; C = coding strand.
\end{tabular}

\section{Culture and cell transfection}

MDCC-MSB1 cells were maintained at $37^{\circ} \mathrm{C}$ in MEM containing $10 \%$ fetal calf serum, 100 $\mathrm{U} / \mathrm{mL}$ penicillin, and streptomycin. Lipofectamine 2000 (Invitrogen, USA) was used to transfect cells with siRNAs. MDCC-MSB1 cells $\left(1.0 \times 10^{7}\right.$ cells $\left./ \mathrm{mL}\right)$ were placed in culture vials without serum or BsAbs 1 day before transfection; the cells were cultured overnight at $37^{\circ} \mathrm{C}$. shRNAs and Lipofectamine 2000 were mixed and incubated at room temperature for 15 min before being used to transfect MDCC-MSB1 cells. Serum-containing medium was added $6 \mathrm{~h}$ later.

\section{Assessing chTERT mRNA expression by RT-PCR}

Glyceraldehyde-3-phosphate dehydrogenase (GAPDH) was used as the reference gene. Primers and probes for RT-PCR were designed using the sequences of GAPDH and chTERT (AY502592) (Table 2). Cells were sampled at 48, 72, and $96 \mathrm{~h}$ after transfection and their RNAs were collected for RT-PCR. A randomly chosen RT-PCR product was used as template and the forward and reverse primers, dNTP, and sterile water were added to attain a total volume of $25 \mu \mathrm{L}$. The amplification conditions were as follows: denaturation at $94^{\circ} \mathrm{C}$ for 2 min, and 40 cycles of $94^{\circ} \mathrm{C}$ for $10 \mathrm{~s}$ and $60^{\circ} \mathrm{C}$ for $31 \mathrm{~s}$. Then, $2 \mu \mathrm{L}$ reverse-transcription products was used as a template and RT-PCR forward and reverse primers and probes were added.

\section{Enzyme activity assay}

Telomerase activity was detected using a modified telomeric repeat amplification protocol (TRAP) approach. The primers were designed using a program written in our laboratory and tested with DNAStar. The sequences of the primers were P1: 5'-TCACCTTGTAATCCGTCGAGCAGAGTT- 
3', and P2: 5'-CAACATCTCCACTACCTTCTAACCGTAACC-3'. For P1, the 3'-end 18-nt sequence was a standard TRAP reaction TS primer, and the 5'-end 9-nt was a random sequence. For P2, the $3^{\prime}$-end was a telomerase sequence (5'-CTAACCGTAACC-3'), while the 5'-end was an anchor end (5'-CAACATCTCCACTACCTT-3'). The telomerase template R5 was equivalent to the P1 5 telomerase repeats catalyzed by telomerase, the sequence being [5'-TCACCTTGTAATCCGTCGAG

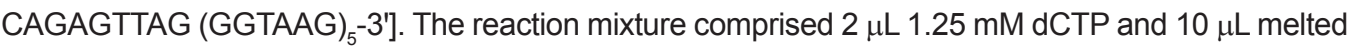
paraffin wax; when the paraffin wax coagulated, $5 \mu \mathrm{L}$ 10X TRAP buffer solution (200 mM Tris- $\mathrm{HCl}$, $\mathrm{pH}$ 8.3., $15 \mathrm{mM} \mathrm{MgCl}_{2}, 0.1 \%$ Tween 220, $630 \mathrm{mM} \mathrm{KCl}, 10 \mathrm{mM}$ EDTA), $50 \mu \mathrm{M}$ dATP, dTTP and dGTP, $15 \mathrm{pmol} / \mu \mathrm{L}$ primers $\mathrm{P} 1$ and P2, $2 \mathrm{U}$ polymerase Ex-Taq, and $2 \mu \mathrm{L}$ sample cells were added, along with sterile water to make up the total volume to $50 \mu \mathrm{L}$. The reaction was allowed to run for $20 \mathrm{~min}$ to synthesize the telomerase templates, then it was heated at $90^{\circ} \mathrm{C}$ for $3 \mathrm{~min}$ to inactivate telomerase, and then PCR-amplified as follows: 30 cycles of $90^{\circ} \mathrm{C}$ for $30 \mathrm{~s}, 64^{\circ} \mathrm{C}$ for $45 \mathrm{~s}$, and $72^{\circ} \mathrm{C}$ for $1 \mathrm{~min}$. The amplification products were separated on native-PAGE and silver-stained.

Table 2. Primers and probes for quantitative real-time PCR.
\begin{tabular}{l|l}
\hline Gene & Primers and probes \\
\hline chTERT & Forward: 5'-GTCAGAGCGAAGTCATCACAAGAAT-3' \\
\cline { 2 - 2 } & Reverse: 5'-TGGCAAAACTCTGAAGTGACAAC-3' \\
\cline { 2 - 2 } & Probe: FAM-ATGGATACTCCTTGCTGGATGAGAA-ECLIPSE \\
\hline \multirow{2}{*}{ GAPDH } & Forward: 5'-TTGTTTCCTGGTATGACAATGAGTTT-3' \\
\cline { 2 - 2 } & Reverse: 5'-CTCACTCCTTGGATGCCATGT-3' \\
\cline { 2 - 2 } & Probe: FAM-ATACAGCAACCGTGTTGTGGACTTGATGGT-ECLIPSE \\
\hline
\end{tabular}

\section{Cell cycle detection}

Cells transfected with pSi-U6-Ctrl and pSi-U6-sh-3 were collected after $72 \mathrm{~h}$. The cells were centrifuged at $1000 \mathrm{rpm}$ for $5 \mathrm{~min}$, washed three times with PBS, and fix in cold $70 \%$ ethanol at $4^{\circ} \mathrm{C}$ for no less than $18 \mathrm{~h}$. The cells were then centrifuged at $1000 \mathrm{rpm}$ for $5 \mathrm{~min}$, washed with PBS, and resuspended in $200 \mu \mathrm{L}$ propidium iodide and RNase (final concentrations of propidium iodide and RNase were 50 and $100 \mathrm{mg} / \mathrm{mL}$, respectively) in the dark for $30 \mathrm{~min}$. A flow cytometer (FACScan, Becton Dickinson, Franklin Lakes, NJ, USA) was used to analyze the cell cycle.

\section{RESULTS}

\section{Construction of the expression vector for pSi-U6-shRNAs}

As described above, double-stranded DNAs were obtained by annealing synthesized DNA into the vector pSilencer 1.0-U6 and transforming the plasmids into E. coli cells. The plasmids were then sequenced to confirm the DNA sequences (Figures 1 and 2). Positive plasmids were named pSi-U6-Ctrl, pSi-U6-sh-1, pSi-U6-sh-2, and pSi-U6-sh-3.

\section{Identification of chTERT and GAPDH clone}

RNAs from MDCC-MSB1 cells were amplified using specific primers and two fragments of the expected size were obtained. The fragments were then ligated into vector pMD18-T and transformed into the competent bacterial strain DH5a. PCR was used to confirm that the bacteria 
carried the two fragments (Figure 3). Two bacterial clones were sent to Dalian Bioengineering, Ltd., for sequencing. A BLASTn analysis showed the recombinant plasmids chTERT FR (119 bp) and GAPDH FR of (97 bp) had 100\% identity with the sequences reported by Li et al. (2014).

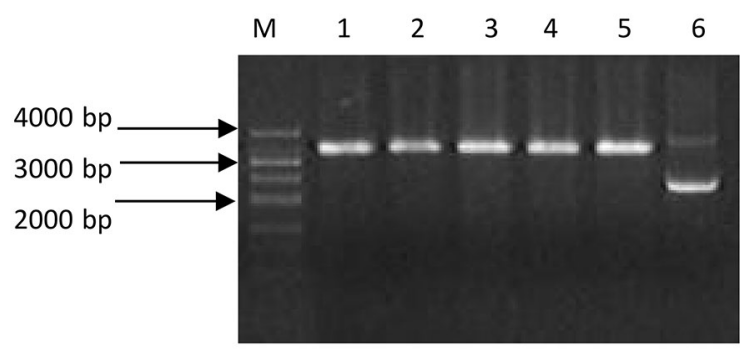

Figure 1. Identification of pSi-U6-sh-1 after digestion with Xhol. Lane $M=$ DL4000 marker; lane 1 = pSi-U6-Ctrl; lane 2 = pSi-U6-sh-1; lane 3 = pSi-U6-sh-2; lane 4 = pSi-U6-sh-3; lane 5 = pSi-U6-Ctrl; lane 6 = positive plasmid.

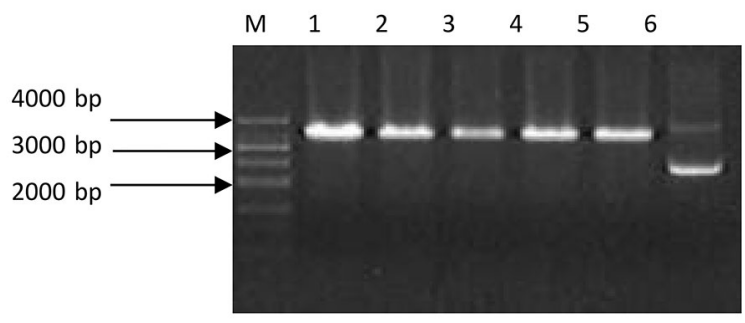

Figure 2. Identification of pSi-U6-sh-1 after digestion with HindIII. Lane M = DL4000 marker; lane 1 = pSi-U6-Ctrl; lane 2 = pSi-U6-sh-1; lane 3 = pSi-U6-sh-2; lane 4 = pSi-U6-sh-3; lane $5=$ pSi-U6-Ctrl; lane $6=$ positive plasmid.

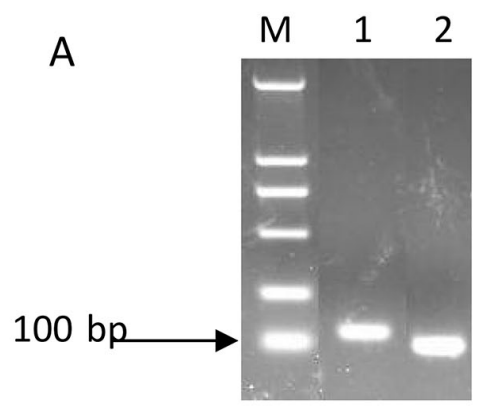

B

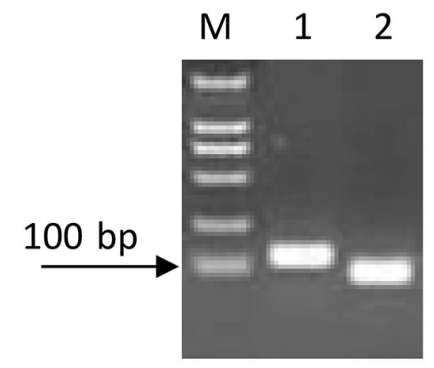

Figure 3. A. PCR products of $C h T E R T$ and GAPDH. B. Identification of recombinant plasmid by PCR amplification. Lane $M=$ DL2000 marker; lane 1 = chTERT; lane 2 = GAPDH.

\section{Inhibition of chTERT mRNA expression by shRNAs}

At $72 \mathrm{~h}$, cells transfected with pSi-U6-sh-3 showed the greatest inhibition (89\%) of chTERT expression (Figure 4). Cells transfected with pSi-U6-sh-1 also showed a significant inhibition of chTERT expression. No effect was observed for pSi-U6-sh-2. An inhibitory effect was evident at 48 $\mathrm{h}$ after transfection, and was still observed at $96 \mathrm{~h}$ (Figure 4). 


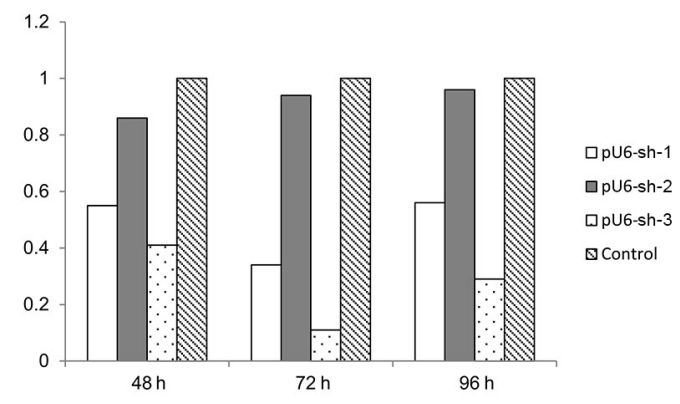

Figure 4. Inhibition of chTERT mRNA expression by shRNA.

\section{Test of telomerase activity}

A modified TRAP method was used to detect telomerase activity. At $48 \mathrm{~h}$ after transfection, there was no evidence of change in telomerase activity in any group. However, in comparison with the control, telomerase activity fell significantly in the pSi-U6-sh-1 and pSi-U6-sh-3 transfected cells at $72 \mathrm{~h}$. The results clearly demonstrated that inhibition of chTERT mRNA expression effectively reduced telomerase activity.

\section{Cell cycle analysis}

Flow cytometry analysis showed that cells transfected with pSi-U6-sh-1 or pSi-U6-sh-3 failed to reach $S$ phase, and that their G2/M periods lengthened to $72 \mathrm{~h}$ after transfection, in contrast with the control group (Table 3). This is a clear evidence that cell division was inhibited. No significant cell cycle changes were observed before $72 \mathrm{~h}$ transfection. No clear cell cycle changes were observed for cells transfected with pSi-U6-sh-2.

Table 3. Cell cycle changes at $72 \mathrm{~h}$ after transfection with pU6-sh-3.

\begin{tabular}{l|c|c|c}
\hline Treatment group & G0/G1 $(\% ;$ mean \pm SD) & G2/M (\%; mean \pm SD) & S (\%; mean \pm SD) \\
\hline Ctrl siRNA & $42.73 \pm 1.57$ & $22.86 \pm 1.16$ & $34.41 \pm 3.22$ \\
\hline siRNA-3 & $44.9 \pm 3.09$ & $51.54 \pm 2.36^{*}$ & $3.56 \pm 0.8^{\star \star}$ \\
\hline
\end{tabular}

${ }^{*} P<0.05 ;{ }^{* *} P<0.01$.

\section{DISCUSSION}

\section{siRNA synthesis}

The phenomenon of RNAi has received considerable attention since it was discovered and it has become one of the most promising methods for treatment of viral infection (Adelman et al., 2001; Yokota et al., 2003; Kahana et al., 2004) At present, a range of approaches for siRNA are available, such as chemical synthesis, external transcription, long dsRNA fragment digestion by RNase III (such as Dicer, E. coli. RNase III), and siRNA expression or viral vector expression in cells. Artificially synthesized or externally transcribed siRNAs are not suitable for the examination of long-term effects in cells because they have a relatively limited lifetime (Brummelkamp et al., $2002 a, b)$. In contrast, the inhibition of target genes by siRNA in expression vectors driven by the 
polymerase III promoters U6 or H1 offer a longer-term approach (Myslinski et al., 2001; Miyagishi and Taira, 2002).

\section{Influence of the target gene local structure}

In RNAi experiments, the selection of the target site for siRNA is very important. The extent of inhibition of the target gene can vary greatly by siRNAs with differences of only a few bases. To date, selection of the interference site on the target gene is still an empirical process, as it is difficult to predict the silencing effect of a given siRNA on a target gene (Kretschmer-Kazemi Far and Sczakiel, 2003). siRNA efficiency may be dependent on the target gene structure, or the ability to access the target gene; this was supported by the results of our study (Kretschmer-Kazemi Far and Sczakiel, 2003). Analysis with M-fold showed a circular structure at the siRNA-3 and siRNA-1 interference sites and a hairpin structure at the siRNA-2 interference site (Zuker, 2003; Gao et al., 2005). Our results showed that different structures at the interference site might produce different inhibition effects. These differences are also reflected in the free activation energies (Table 4). siRNA-2 had the highest free activation energy and the least interference effect, while the reverse was true for shRNA-1 and shRNA-3.

Table 4. Features of the siRNAs for chTERT mRNAs.

\begin{tabular}{l|l|c|l|c}
\hline siRNA & Sequence of SiRNA & Location & Structure of RNA & $\rho \mathrm{G}(\mathrm{kcal} / \mathrm{mol})$ \\
\hline siRNA-1 & AAGGTCTGGTTATCAGCCTTC & $835-855$ & circular & -3.6 \\
\hline siRNA-2 & AAGCCATAACAAATGCCGGTTC & $2104-2124$ & hairpin & -0.2 \\
\hline SiRNA-3 & AAGATCCCAAGGGATACTATG & $3995-4015$ & circular & -4.7 \\
\hline
\end{tabular}

\section{Analysis of siRNA interference effect}

mRNA profile and target gene expression are used to demonstrate an siRNA inhibitory effect. Semi-quantitative RT-PCR, real-time PCR, and northern hybridization are widely used for mRNA assays and the silencing effect is judged by signal strength. RT-PCR (also known as TaqMan Probing) is generally accepted and is the most precise technique for mRNA expression studies (Sasaki et al., 2014). All mRNA copies are counted in RT-PCR, which contains relative quantification and absolute quantification. Some researchers believe that the factors affecting PCR amplification cannot be detected, which is an important problem for quantification (Kellner et al., 2014). Relative quantification refers to determination of expression of the target gene compared to that of a reference gene; since the level of expression of the reference gene is constant, it can be used for comparison of expression differences from different sources. Interfering factors like impurities or leftover reagents can be eliminated by mathematical calculation; therefore, the method is simple, fast and reliable (Chapman et al., 2012). Therefore, to ensure accuracy of our results, we used a relative quantification approach with GAPDH as the internal reference gene to determine the amount of chTERT mRNA in the sample.

\section{Role of chTERT in MDCC-MSB1 cells}

TERT is an important part of the telomerase complex that catalyzes the repeat amplification of telomeric DNA sequences (Xie et al., 2014). TERT is the template for amplification because of its constant expression level in most normal cells. The expression of TERT increases in immortalized 
cells (Xie et al., 2014). The telomeric sequences in $90 \%$ of tumor cells are much shorter than those in normal cells, indicating that TERT has a role in maintaining telomere length and, thus, a stable chromosome (Xie et al., 2014). Our study confirmed that shRNAs were expressed by siRNA expression vectors transfected into MDCC-MSB1 cells using U6 as the promoter. At $72 \mathrm{~h}$ after transfection, up to $89 \%$ of chTERT mRNA expression was inhibited, telomerase activity was greatly reduced, and the proportion of cells in $S$ phase reduced remarkably $(P<0.01)$.

To summarize, our research proved conclusively that siRNAs can effectively inhibit the expression of chTERT mRNA in MDCC-MSB1 cells, reducing telomerase activity, and prevent MDCC-MSB1 cells from entering S phase. Thus, the siRNAs prevent proliferation of MDCC-MSB1 cells. Our study provides information that will be of value for future studies and may facilitate research into cancer treatments based on siRNA technology.

\section{Conflicts of interest}

The authors declare no conflict of interest.

\section{ACKNOWLEDGMENTS}

Research supported by the National Natural Science Fund.

\section{REFERENCES}

Adelman ZN, Blair CD, Carlson JO, Beaty BJ, et al. (2001). Sindbis virus-induced silencing of dengue viruses in mosquitoes. Insect Mol. Biol. 10: 265-273. http://dx.doi.org/10.1046/j.1365-2583.2001.00267.x

Brummelkamp TR, Bernards R and Agami R (2002a). A system for stable expression of short interfering RNAs in mammalian cells. Science 296: 550-553. http://dx.doi.org/10.1126/science.1068999

Brummelkamp TR, Bernards R and Agami R (2002b). Stable suppression of tumorigenicity by virus-mediated RNA interference. Cancer Cell 2: 243-247. http://dx.doi.org/10.1016/S1535-6108(02)00122-8

Chapman HM, Schutt KL, Dieter EM and Lamos SM (2012). Relative quantification of biomarkers using mixed-isotope labeling coupled with MS. Bioanalysis 4: 2525-2541. http://dx.doi.org/10.4155/bio.12.208

Delany ME and Daniels LM (2004). The chicken telomerase reverse transcriptase (chTERT): molecular and cytogenetic characterization with a comparative analysis. Gene 339: 61-69. http://dx.doi.org/10.1016/j.gene.2004.05.024

Delecluse HJ and Hammerschmidt W (1993). Status of Marek's disease virus in established lymphoma cell lines: herpes virus integration is common. J. Virol. 67: 82-92.

Gao L, Zhang L, Hu J, Li F, et al. (2005). Down-regulation of signal transducer and activator of transcription 3 expression using vector-based small interfering RNAs suppresses growth of human prostate tumor in vivo. Clin. Cancer Res. 11: 63336341. http://dx.doi.org/10.1158/1078-0432.CCR-05-0148

Kahana R, Kuznetzova L, Rogel A, Shemesh M, et al. (2004) Inhibition of foot-and-mouth disease virus replication by small interfering RNA. J. Gen. Virol. 85: 3213-3217. http://dx.doi.org/10.1099/vir.0.80133-0

Kellner S, Ochel A, Thüring K, Spenkuch F, et al. (2014). Absolute and relative quantification of RNA modifications via biosynthetic isotopomers. Nucleic Acids Res. 42: e142. http://dx.doi.org/10.1093/nar/gku733

Kretschmer-Kazemi Far R and Sczakiel G (2003). The activity of siRNA in mammalian cells is related to structural target accessibility: a comparison with antisense oligonucleotides. Nucleic Acids Res. 31: 4417-4424. http://dx.doi.org/10.1093/nar/gkg649

Li YH, Liu XM, Yang Z, Xu CG, et al. (2014). The MYC, TERT, and ZIC1 genes are common targets of viral integration and transcriptional deregulation in avian leukosis virus subgroup J-induced myeloid leukosis. J. Virol. 88: 3182-3191.

Miyagishi M and Taira K (2002). U6 promoter-driven siRNAs with four uridine 3' overhangs efficiently suppress targeted gene expression in mammalian cells. Nat. Biotechnol. 20: 497-500. http://dx.doi.org/10.1038/nbt0502-497

Myslinski E, Amé JC, Krol A and Carbon P (2001). An unusually compact external promoter for RNA polymerase III transcription of the human H1RNA gene. Nucleic Acids Res. 29: 2502-2509. http://dx.doi.org/10.1093/nar/29.12.2502 
Olejniczak M, Galka P and Krzyzosiak WJ (2010). Sequence-non-specific effects of RNA interference triggers and microRNA regulators. Nucleic Acids Res. 38: 1-16. http://dx.doi.org/10.1093/nar/gkp829

Pecot CV, Calin GA, Coleman RL, Lopez-Berestein G, et al. (2011). RNA interference in the clinic: challenges and future directions. Nat. Rev. Cancer 11: 59-67. http://dx.doi.org/10.1038/nrc2966

Peng W, Feng M, Feng X, Liang YX, et al. (2015). An archaeal CRISPR type III-B system exhibiting distinctive RNA targeting features and mediating dual RNA and DNA interference. Nucleic Acids Res. 43: 406-417. http://dx.doi.org/10.1093/nar/gku1302

Sasaki T, Ogose A, Kawashima H, Hotta T, et al. (2014). Real-time polymerase chain reaction analysis of MDM2 and CDK4 expression using total RNA from core-needle biopsies is useful for diagnosing adipocytic tumors. BMC Cancer 14: 468. http://dx.doi.org/10.1186/1471-2407-14-468

Swanberg SE, Payne WS, Hunt HD, Dodgson JB, et al. (2004). Telomerase activity and differential expression of telomerase genes and c-myc in chicken cells in vitro. Dev. Dyn. 231: 14-21. http://dx.doi.org/10.1002/dvdy.20149

Venkatesan RN and Price C (1998). Telomerase expression in chickens: constitutive activity in somatic tissues and downregulation in culture. Proc. Natl. Acad. Sci. USA 95: 14763-14768. http://dx.doi.org/10.1073/pnas.95.25.14763

Woźniakowski G and Niczyporuk JS (2015). Detection of specific UL49 sequences of Marek's disease virus CVI988/Rispens strain using loop-mediated isothermal amplification. J. Virol. Methods 221: 22-28 http://dx.doi.org/10.1016/j.jviromet.2015.04.015

Xie H, Liu T, Wang N, Björnhagen V, et al. (2014). TERT promoter mutations and gene amplification: promoting TERT expression in Merkel cell carcinoma. Oncotarget 5: 10048-10057. http://dx.doi.org/10.18632/oncotarget.2491

Yokota T, Sakamoto N, Enomoto N, Tanabe Y, et al. (2003). Inhibition of intracellular hepatitis C virus replication by synthetic and vector-derived small interfering RNAs. EMBO Rep. 4: 602-608. http://dx.doi.org/10.1038/sj.embor.embor840

Zuker M (2003). Mfold web server for nucleic acid folding and hybridization prediction. Nucleic Acids Res. 31: 3406-3415. http://dx.doi.org/10.1093/nar/gkg595 